\section{Prediction rule}

\section{CT should not be relied on for cases of isolated vomiting in children with blunt head trauma}

10.1136/ebmed-2014-110059

\section{Thierry A G M Huisman}

Department of Radiology and Radiological Science, Johns Hopkins Hospital, Baltimore, Maryland, USA

Correspondence to: $\mathrm{Dr}$ Thierry A G M Huisman, Department of Radiology and Radiological Science, Johns Hopkins Hospital 1800 Orleans Street, Suite 4174, Baltimore, MD 21287-0842, USA; thuisma1@jhmi.edu

Commentary on: Dayan PS, Holmes JF, Atabaki S, et al. Association of traumatic brain injuries with vomiting in children with blunt head trauma. Ann Emerg Med 2014;63:657-65.

\section{Context}

Accurate emergency diagnostic work up of children who have sustained a minor traumatic brain injury (TBI) is challenging. In most cases, symptoms are non-specific and may include episodes of altered or transient loss of consciousness, irritability, sleepiness or headache. Each physician must decide if clinical observation is sufficient to exclude intracranial complications or if a more aggressive diagnostic approach, including neuroimaging (eg, CT) should be pursued. The growing awareness of radiation-induced side effects urges each physician to take full advantage of the clinical findings. The current paper suggests that a history of vomiting is linked to an increased prevalence of TBI seen on CT, while the absence of vomiting is coupled with a decreased prevalence of TBI on CT. The desire to have an easy, safe, practical and reproducible diagnostic sign to predict TBI may have pushed for the inclusion of 'vomiting' in the diagnostic algorithm. The authors of this paper studied the significance and characteristics of vomiting in children with minor TBI.

\section{Methods}

A secondary analysis of a large prospective observational study conducted at 25 centres was performed. Inclusion criteria were: age $\leq 18$ years; blunt head trauma, with presentation to the emergency department (ED) after $<24 \mathrm{~h}$ of trauma; Glasgow Coma Scale score of 14-15; and acute head CT. Clinicians completed a standardised patient history and physical examination before head CT. History of vomiting, the number of vomiting episodes, timing of onset and the time since last episode were recorded. Isolated vomiting was defined in two ways: extensive (based on an extensive list of variables) versus an age-specific list of prediction rule variables defined by the Pediatric Emergency Care Applied Research Network (PECARN) on initial ED examination. Two categories of outcomes were defined: clinically-important TBI and TBI on CT. Outcomes were analysed in three groups: children with non-isolated vomiting; children with isolated vomiting (extensive definition); and children with isolated vomiting (PECARN definition). The rates of clinically-important TBI and TBI on CT were compared for children with and without isolated vomiting using the Newcombe-Wilson continuity-adjusted method.
Findings

Of 43904 enrolled patients, 5392 children were included in the study. A total of 4577 (84.9\%) had non-isolated vomiting, while 815 (15.1\%) had isolated vomiting. Head CTs were performed in 3284 children (71.8\%) with non-isolated vomiting and in 298 (36.6\%) children with isolated vomiting. All patients with vomiting and clinically-important TBI had TBI on CT. Patients with isolated vomiting had a low prevalence of clinically-important TBI. Clinically-important TBI occurred in 2 of 815 $(0.2 \%)$ patients in the isolated vomiting group, versus 114 of 4577 (2.5\%) in the non-isolated vomiting group. TBI on CT occurred in 5 of 298 (1.7\%) patients in the isolated vomiting group versus 211 of 3284 (6.4\%) in the non-isolated vomiting group.

The authors conclude that TBI on CT is uncommon and clinically-important TBI is very uncommon in children when vomiting is the only clinical symptom. CT is consequently not required in these children; however, they should be observed for progression of symptoms. TBI is more frequent in children when vomiting is accompanied by other signs suggestive of TBI. In these children head CT should be considered.

\section{Commentary}

This study evaluates an easy-to-collect clinical marker (isolated vomiting) for risk stratification of intracranial lesions in paediatric blunt TBI. The authors conclude that clinically-important brain injury is rarely present in children with TBI when vomiting is the only clinical sign. Consequently, the authors suggest that head CT is generally not required. I partially disagree with this statement. It may be true that no findings are to be expected that require emergent neurosurgical treatment in this setting; however, a negative CT does not mean that the brain is not injured-instead the injury may not be visible on the acute CT. It is well known that CT may underestimate the degree of TBI. ${ }^{1}$ MRI may identify TBI, while CT appears unremarkable. In particular, diffuse axonal injury may only be seen on advanced sequences such as susceptibility-weighted or diffusion-weighted imaging. In addition, ${ }^{1} \mathrm{H}$ MR spectroscopy, diffusion tensor imaging or functional MRI may reveal diffuse injury to the neuroarchitecture and neurofunction. This injury may have an impact on acute neurocognition or neurofunctionality, as well as ongoing and future paediatric brain development, which may remain undetected on acute clinical evaluation, especially if the child is very young. Neurobehavioural tests and follow-up multimodality MRI should be considered if the child has persisting symptoms.

Controversy about potentially harmful exposure to ionising radiation (CT) should not blind us to the possible long-lasting effects of diffuse brain injury that remained undetected by CT. This may delay or prevent neuroprotective or neurostimulation treatments. Whenever possible and indicated, MRI should also be considered in the emergent diagnostic work up. Ultrafast, highly sensitive and specific MR protocols can give valuable information about outcome, can guide and validate acute and long-term treatment and help parents in their decision-making in combination with the clinical data.

\section{Competing interests None.}

Provenance and peer review Commissioned; internally peer reviewed.

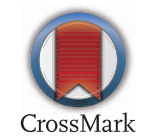

\section{Reference}

1. Ashwal S, Tong KA, Ghosh N, et al. Application of advanced neuroimaging modalities in pediatric traumatic brain injury. J Child Neurol 2014;22:pii: 0883073814538504 